\title{
Editorial \\ Socioeconomic assessment of diet on health and quality of life
}

\section{Introduction}

Across the lifespan of a human being, diet plays an important role in their growth, development and health. Food supplies energy for the functioning of vital activities such as metabolism and homeostasis. Generally, unhealthy food choices are associated with hectic work schedules, and busy lifestyles have led to diffusion of several lifestyle-related chronic health diseases. An adequate nutrient regime can decrease the incidence of many diseases and specific deficiencies such as metabolic syndrome, type 2 diabetes mellitus, cardiovascular diseases, cancer, infection, cognitive impairment, poor quality of life, disability and mortality (Jayanama et al., 2021). On the contrary, poor nutritional habits can lead to high incidence of disease and high social costs (Sharma et al., 2021). For instance, obesity is strongly associated to low life expectancy and quality of life. However, this food disorder affects also the social life of patients because they often are unemployed or have social disadvantages or reduced socioeconomic productivity. In Europe, poor people have a greater risk (ranging from 10 to $20 \%$ ) of being obese than people in high income categories (Amenyah et al., 2021).

Therefore, the theme of correct nutritional regime is crucial in all the political agendas elaborated in the European and international context. For implementing these actions, it is fundamental to know how much this phenomenon is spread worldwide and measure it. Nevertheless, to prevent diseases it is important not only to consider the nutrients intake, but the dietary quality pattern. Currently, public opinion is subject to an insistent awareness campaign by the governments aiming at making known what are the main consequences for unhealthy food choices. For instance, the Mediterranean diet is advised worldwide since it is a balanced dietary pattern characterized principally by vegetables, olive oil and pasta intake. Studies, in fact, have underlined its awesome capacity of reducing the incidence of mortality rate from cardiovascular diseases, cancers, and diabetes and improving the life span in older adults (Jayanama et al., 2021; Fiore et al., 2019; Spada et al., 2020).

The nutritional patterns of a population can act as an indicator for understanding the level of progress of a country. Strengthening and promoting the commitment of health food systems is the new and innovative strategy carried out by the governments to improve population income, food security and quality life (WHO, 2018). In this context a correct nutrition is one of the cornerstones of preventive and curative medicine (Singh et al., 2017). Moreover, it is believed that better health outcomes can be achieved for each euro spent guaranteeing the population an adequate nutritional intake (Galekop et al., 2021). The report published by the International Obesity Task Force shows that malnutrition in Europe is constantly increasing and, in some areas, it reaches the same percentages registered in the USA (Lobstein et al., 2005). However, the determination of the economic impact remains the most complex part of the general assessments. In particular, the topic of "socioeconomic assessment of diet on health and quality of life" is still being explored by governments, economists, practitioners, industry and producers due to its complexity. Research, practice, EU policies and classroom teaching have to aim at focusing on discussion of issues related to behavior of firms, consumers and politicians within these main trends that otherwise would tend to remain implicit and unspoken.

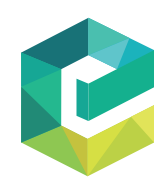

British Food Journal Vol. 123 No. 8,2021 pp. $2617-262$

(c) Emerald Publishing Limited 0007-070X 
$\mathrm{BFJ}$

123,8

2618

In light of the growing interest in the potential benefit that society might have from integrating the health habits into food choices, it is believed that this special issue (SI) can be useful to give new insights on these global questions. Thus, this SI has encouraged researchers in studying innovative approaches to submit their ideas. Therefore, the present SI offers an overview on models, definitions and concepts associated with health and nutrition in order to provide industry, researchers, teachers and students new tools to extend perspectives of food systems.

\section{An overview on papers presented in the special issue}

The papers collected in the present SI deal with different points of view, perspectives and carry out various methodologies. Indeed, most of the papers highlight the importance of a healthy diet and food education, paying attention to eating habits of young consumers, who often neglect in their diet fruits and vegetables. The issue of organic food was also addressed, in the light of changes in supply and demand in this market, in which it was found that often the only reasons that lead to the purchase of the organic product are health-related. Also, the impact of quality and sustainability labeling was examined in relation to its perception of a typical product of the Mediterranean diet (i.e. olive oil), but this approach certainly can be extended as a research line for other products. Furthermore, new methodological frontiers have been used to address the issue of functional nutrition, especially in reference to the elderly population. Besides, the evaluation of public food procurement was also addressed in the context of the short supply chain and organic food, bridging the knowledge gap present until now. Even the measurement of food waste, a subject now very dear to the world scientific literature, was addressed not only from a quantitative point of view but by giving a strong weight to the ethical aspect of the problem.

In particular, the paper "Does organic means health for consumers? Selected issues of organic food market" by investigating statistical data available on organic food markets in France and Poland, identifies what the main drivers of consumer choices are and how the supply answers the increasing demand for organic food. Findings highlight as the health properties of organic food represent crucial factors affecting organic food purchasing. In line with the latter's topic, the paper "Impact of organic and "protected designation of origin" labels in the perception of olive oil sensory quality", carrying out an internet-based interview, investigates behavior and habits of the Italian consumers of extra virgin olive oil, focusing on the impact of Protected Designation of Origin (PDO) and EU organic certification.

Another work from the consumers' perspective is titled "Socio-demographic influences on various consumer food safety indices: an empirical study of ethnic Goan pork sausage consumers"; its aim is to measure choice behavior and health hazard concerns of consumers of ethnic Goan pork sausage by using food safety indices; results show younger people with high education and higher income are the most prevalent consumers.

The latter is investigated in the work "Young consumers' perception of food safety and their trust in official food control agencies" that has the goal of understanding how young Poland consumers perceive food safety and if they trust the role of official food control agencies. Findings highlight that young people do not refer to food safety in a correct and complete meaning as expressed in the EU and national regulations and strategies.

On the other hand, in "Clustering Generation Z university students based on daily fruit and vegetable consumption: empirical research in an emerging market", the authors, thanks to administrating a survey, demonstrate the existence of a choice behavior of Romanian Generation $Z$ members toward a healthy and nutritional lifestyle based primarily on the consumption of fresh vegetables and fruit.

The importance and the role of health food is also analyzed in the work "Local food communities: exploring health-related adaptivity and self-management practices" that, by 
means of a qualitative research approach, gives evidence of the importance of local food communities to promote own potential for health-food models and to improve understanding of the mechanisms that support a healthy diet. In line with this context, the work "Dynamic conceptual framework to investigate adoption of healthy diet through agent-based modelling" tries to build up a dynamic conceptual framework based on an agent-based model where intention represents the key driver of the tendency to adopt a healthy diet. The connection between intention and behavior is made dynamically balanced by perceived control factors (price, accessibility to healthy food, time).

The role of education for healthy choices is also dealt with. Indeed, the paper "The effect of education on fruit and vegetable purchase disparities in Chile" analyzes by means of OaxacaBlinder decomposition of fruit and vegetables purchases and expenditure in Chile in the last five years by the level of household head education. Findings show how decomposing the effect of education can contribute to focus on initiatives that can provide nutrition and health disparities. Indeed, changes in food environment conditions appear to be more likely to explain changes on fruit and vegetables purchase disparities compared to the changes in the education of the household head.

Focusing on the role of an educator is the starting point of the paper "A vegetarian educator in a meat obsessed country" that deals with four emerging themes: vegetarian experience, culture, identity as an educator and impacts of beliefs to address the experience of living in a meat-based culture and the emotions related to the lifestyle choice. Finally, a vegetarian has to overcome several obstacles to support own identity when social group can believe this lifestyle untraditional.

Another important role to address the food choices is certainly played by the marketing strategies: the work "The effects of advertisements on consumer choices and health: a content analysis of health claims in Italian magazines" has the purpose of analyzing food advertising in Italian magazines based on the content analysis methodological approach. The main findings coming out are the necessity to communicate with a superior degree of precision not only the sort of product and its characteristics but above all the relative health benefits.

Functional food was addressed within the study of awareness, knowledge and consumption pattern among Indian consumers in the work "Consumer awareness and willingness to purchase probiotic food and beverage products: a study of Sonipat District, Haryana". The paper shows changing customer attitudes as a higher proportion of the population is interested in probiotic products delivered with fruit as a base matrix followed by dairy products. However, government initiation and support are required both for manufacturing such products as well as spreading awareness on the necessity of including them in the meals of the growing children.

A current topic of game meat consumption is investigated within the paper "Differences in game consumption patterns between North and South China". The regional approach applied allows to conclude that consumers in the southern region have a preference for wildife because of such factors as natural environment, history and culture, and the level of economic development. Even if game meat is not a major component of the daily diet, the government should consider banning this trade to prevent future disease outbreaks caused by wildlife-tohuman transmissions.

Functional food ingredients were addressed in the work "Grape residue flour as an antioxidant and fiber source in beef meatballs", which proves that the addition of grape flours reduces the lipid oxidation of meatballs by close to $50 \%$ compared to the control sample. It allows for decrease in the addition of synthetic additives, adds fiber to the consumer's diet and prevents inappropriate waste disposal. The wine sector is also addressed regarding methods to assess the authenticity and traceability of wines allowing producers to obtain market recognition and premium prices. The paper "Derivatized volatile organic compound characterization of Friulano wine from Collio (Italy-Slovenia) by HS-SPME-GC-MS and 
$\mathrm{BFJ}$ 123,8

discrimination from other varieties by chemometrics" proposes a two-step chemometric approach based on an unsupervised technique (Principal Component Analysis (PCA)) followed by a supervised one (Partial Least-Squares Discriminant Analysis (PLS-DA)). The results confirm the benefits of using a derivatization step prior to volatile organic compounds analysis.

Some health risk regarding possible initiation of cancer because of radiation is a topic presented in the work "Preliminary survey of ${ }^{226} \mathrm{Ra},{ }^{232} \mathrm{Th}$ and ${ }^{40} \mathrm{~K}$ activity level and their cancer risk in some foodstuff, Sudan". This study introduces some baseline information for radiation food safety in view of radiation protection. A different disease risk is addressed by the paper "The longitudinal association between soybean and non-soybean legumes intakes and risk of cardiovascular disease: Isfahan cohort study". Results achieved suggest that in the long run, even modest consumption of legumes, but not soybeans alone, can be effective to reduce risk of consumption and cardiovascular events in a low-income population.

Health benefits of food are especially important for some consumer segments. The paper "Functional food consumption among older consumers in Malaysia: a Health Belief Model perspective" provides conclusions on the importance of perceived barriers and cue to action but lack of influence in the case of perceived susceptibility in the studied age group.

Food safety can be addressed within one of its basic dimension such as shelf life. This issue is a starting point for a study "Modified atmosphere packaging for milk cake: assessment of ideal gas composition for extending shelf life", whose results prove that Modified Atmosphere Packaging (MAP) is the best among the ways investigated for extending the shelf life of milk cake up to 28 days at refrigeration temperature. The latter paper "Food waste measurement toward a fair, healthy and environmental-friendly food system: a critical review" addresses food safety more in general assuming that food waste measurement is essential to rebalance the actual inadequate food system and to switch it toward a fair, healthy and environmentally friendly one. The study concludes on the lack of standardized methodologies, the limits of those proposed and the deficiency of comparable results to achieve sustainable international goals.

Food and meal services are in a scope of the public sector which is the starting point for the paper "Public food procurement for Italian schools: results from analytical and content analyzes". The study analyzes criteria used for evaluation of public food tenders and provides a methodological framework for assigning scores to their clustering. It concludes that food quality and resources categories have greater importance in the evaluation phase of almost all cases investigated.

\section{Mariantonietta Fiore, Roberto Leonardo Rana, Alessia Spada and}

Nina Drejerska

\section{References}

Amenyah, S.D., Murphy, J. and Fenge, L. (2021), "Evaluation of a health-related intervention to reduce overweight, obesity and increase employment in France and the United Kingdom: a mixedmethods realist evaluation protocol", BMC Public Health, Vol. 21 No. 1.

FAO, IFAD, UNICEF, WFP and WHO (2018), The State of Food Security and Nutrition in the World 2018. Building Climate Resilience for Food Security and Nutrition, FAO, Rome, available at: http://www.fao.org/3/19553en/i9553en.pdf (accessed 29 April 2021).

Fiore, M., Alaimo, L. and Chkhartishvil, N. (2019), "The amazing bond among wine consumption, health and hedonistic well-being”, British Food Journal, Vol. 122 No. 8, pp. 2707-2723.

Galekop, M.M.J., Uyl-de Groot, C.A. and Ken Redekop, W. (2021), "A systematic review of costeffectiveness studies of interventions with a personalized nutrition component in adults", Value in Health, Vol. 24 No. 3, pp. 325-335. 
Jayanama, K., Theou, O., Godin, J., Cahill, L., Shivappa, N., Hébert, J.R., MichaelWirth, D.M.D., Park, Y.M., Fung, T.T. and Rockwood, K. (2021), "Relationship between diet quality scores and the risk of frailty and mortality in adults across a wide age spectrum”, BMC Medicine, Vol. 19 No. 1.

Lobstein, T., Rigby, N. and Leach, R. (2005), EU Platform on Diet Physical Activity and Health: EU Platform Briefing Paper of International Obesity Task Force, European Association for the Study of Obesity, Brussels, p. 34, available at: http://ec.europa.eu/health/ph_determinants/life_ style/nutrition/documents/iotf_en.pdf (accessed 29 April 2021).

Sharma, S., Katoch, V., Kumar, S. and Chatterjee, S. (2021), "Functional relationship of vegetable colors and bioactive compounds: implications in human health", Journal of Nutritional Biochemistry, Vol. 92.

Singh, R., Prasad, K.K., Siddiqui, M.W. and Prasad, K. (2017), "Medicinal plants in preventive and curative roles for various ailments", Plant Secondary Metabolites, Biological and Therapeutic Significance, Vol. 1, pp. 63-100.

Spada, A., Rana, R.L. and Fiore, M. (2020), "Investigating the evocative link among wine consumption, Human Development Index and geographical region", Studies in Agricultural Economics, Vol. 122, pp. 66-76. 